\title{
CREATIVE AND WEAKLY CREATIVE SEQUENCES OF r.e. SETS
}

\author{
v. D. VUCKOVIC
}

1. In [1] Cleave introduced the notion of a creative sequence of r.e. (recursively enumerable) sets and proved that all such sequences are $r$. (recursively) isomorphic and 1-1 universal for the class of all r.e. sequences of r.e. sets. In [2] and [3] Lachlan introduced an alternate definition and proved its equivalency with the definition of Cleave.

A sequence of r.e. sets $E_{0}, E_{1}, \cdots$ is called r.e. iff there is an r. function $g$ such that $E_{i}=w_{g(i)}$ for every $i \in N$, where

$$
x \in w_{i} \leftrightarrow \bigvee_{v} T_{1}(i, x, y) .
$$

Cleave calls a disjoint r.e. sequence $E_{0}, E_{1}, \cdots$ of r.e. sets creative if there is a p. (partial) r. function $f$ such that for every disjoint r.e. sequence $w_{h(i)}, i=0,1, \cdots$, (with recursive $h$ ) satisfying $E_{i} \cap w_{h(i)}$ $=\varnothing$, for all $i$, we have, for every $x \in I(h)$,

$$
f(x) \notin \bigcup_{\mu=0}^{\infty}\left(w_{h(\mu)} \cup E_{\mu}\right) .
$$

$I(h)$ is the set of indices of $h$ in the standard enumeration

$$
\phi_{0}, \phi_{1}, \phi_{2}, \cdots,
$$

of all r.p. functions, i.e.,

$$
\phi_{i}(x) \simeq U\left(\mu_{y} T_{1}(i, x, y)\right) .
$$

Lachlan, in [2], proceeds as follows. Let first $g$ be recursive and such that

$$
\bigvee_{y} T_{2}(i, n, x, y) \leftrightarrow \bigvee_{y} T_{1}(g(i, n), x, y)
$$

Define the double sequence $W_{i, n}$ of r.e. sets by $W_{i, n}=w_{g(i, n)}$.

After Lachlan, an r.e. sequence $E_{0}, E_{1}, \cdots$ of r.e. sets is creative iff there is a recursive $f$ such that for all $i$

$$
W_{i, f(i)} \cup E_{f(i)} \subset \bigcup_{\mu=0}^{\infty}\left(W_{i, \mu} \cap E_{\mu}\right) .
$$

Received by the editors March 1, 1967. 
Both Cleave's and Lachlan's definition seem to demand very much to be satisfied: (1.1) involves all indices $x$ of $h$, and (1.5) all indices $i$ (which are, in essence, indices of all r.e. sequences).

In this paper we propose a very weak definition of a creative sequence and prove its equivalency with the definition of Cleave (and so with the definition of Lachlan). Moreover, our definition is a direct generalization of the corresponding Smullyan's definition of a doubly weakly creative pair (Smullyan $[4$, p. 114]).

2. Obviously, a sequence $A_{0}, A_{1}, \cdots$ of r.e. sets is r.e. iff the predicate $x \in A_{y}$ is r.e. Let $\gamma$ be recursive and such that

$$
\bigvee_{v} T_{2}(u, \mu, x, y) \leftrightarrow \bigvee_{v} T_{1}(\gamma(\mu, u), x, y)
$$

For every r.e. predicate $Q(\mu, x)$ there is an $e \in N$ such that $Q(\mu, x)$ $\leftrightarrow \bigvee_{y} T_{2}(e, \mu, x, y)$. With $Q(\mu, x) \leftrightarrow x \in A_{\mu}$ we conclude: every r.e. sequence of r.e. sets can be represented as a sequence $w_{\gamma(\mu, e)}$ $\mu=0,1, \cdots$ for some $e$.

By the recursion theorem, for every r.e. predicate $Q(\mu, z, x, u)$ there is a recursive $\phi$ such that for all $i \in N$,

$$
Q(\mu, i, x, \phi(i)) \leftrightarrow \bigvee_{y} T_{2}(\phi(i), \mu, x, y)
$$

i.e., by (2.1),

$$
Q(\mu, i, x, \phi(i)) \leftrightarrow \bigvee_{\nu} T_{1}(\gamma(\mu, \phi(i)), x, y) .
$$

Lemma 2.1. Let $A_{0}, A_{1}, \cdots$ be an r.e. sequence of r.e. sets and let $f$ be any $r$. function. Then there is an $r$. function $\phi$ such that, for every $i \in N$,

$$
\begin{aligned}
& i \in A_{\mu} \rightarrow w_{\gamma(\mu, \phi(i))}=\{f(\phi(i))\} ; \\
& i \notin A_{\mu} \rightarrow w_{\gamma(\mu, \phi(i))}=\varnothing
\end{aligned}
$$

Proof. ( $\{a\}$ denotes the singleton whose unique element is $a$; $\varnothing$ is the empty set.) In (2.3) take

$$
Q(\mu, z, x, u) \leftrightarrow z \in A_{\mu} \wedge x=f(u) .
$$

From this lemma we obtain immediately.

Lemma 2.2. Let $A_{0}, A_{1}, \cdots$ be a disjoint r.e. sequence of r.e. sets. Then there is an $r$. function $\phi$ such that, for every $i \in N$,

(2.6) $i \in A_{\mu} \rightarrow w_{\gamma(\mu, \phi(i))}=\{f(\phi(i))\}$ and all others $w_{\gamma(\nu, \phi(i))}$ are empty for $\nu \neq \mu$, and

(2.7) $i \notin A_{\mu} \rightarrow$ all $w_{\gamma(\mu, \phi(i))}$ are empty. 
Definition 2.1. An r.e. sequence $A_{0}, A_{1}, \cdots$ of r.e. sets is meager iff either all $A_{\mu}$ are empty or all but one are empty and this one, which is not empty, is a singleton.

Definition 2.2. A disjoint r.e. sequence $A_{0}, A_{1}, \cdots$ of r.e. sets is weakly creative under an $\mathrm{r}$. function $f$ iff, for all $i \in N$ for which the sequence $w_{\gamma(0, i)}, w_{\gamma(1, i)}, \cdots$ is meager,

(a) in case all $w_{\gamma(\mu, i)}$ are empty we have

$$
f(i) \notin \bigcup_{\mu=0}^{\infty} A_{\mu}
$$

(b) in case $w_{\gamma\left(n_{1}, i\right)}$ is not empty and $w_{\gamma\left(n_{0}, i\right)} \cap A_{n_{0}}=\varnothing$, we have

$$
f(i) \notin w_{\gamma\left(n_{0}, i\right)} \text {. }
$$

3. We prove some theorems from which will follow the equivalency of the weak creativity and the creativity in the sense of Cleave.

THEOREM 3.1. If the sequence $E=E_{0}, E_{1}, \cdots$ is weakly creative then every disjoint r.e. sequence $A=A_{0}, A_{1}, \cdots$ of r.e. sets is reducible to $E$.

Proof. Let $E$ be creative under $f$. By Lemma 2.2 there is an $\mathrm{r}$. function $\phi$ such that for every sequence $\Omega_{i}=w_{\gamma(0, \phi(i))}, w_{\gamma(1, \phi(i))}, \cdots$, we have

(3.1) $i \in A_{\mu} \rightarrow \Omega_{i}$ is meager and $w_{\gamma(\mu, \phi(i))}=\{f(\phi(i))\}$, and

(3.2) $i \notin A_{\mu} \rightarrow \Omega_{i}$ is meager and all $w_{\gamma(\mu, \phi(i))}$ are empty.

We shall prove that $\psi=f(\phi)$ reduces $A$ to $E$.

Suppose first that $i \in A_{\mu}$. Then $w_{\gamma(\mu, \phi(i))}=\{f(\phi(i))\}$ and, therefore,

$$
f(\phi(i)) \in w_{\gamma(\mu, \phi(i))} .
$$

If now $w_{\gamma(\mu, \phi(i))} \cap E_{\mu}=\varnothing$ we will have, by (2.9), $f(\phi(i)) \notin w_{\gamma(\mu, \phi(i))}$ in contradiction to (3.3). Therefore, $f(\phi(i)) \in E_{\mu}$.

To prove the opposite inclusion

$$
f(\phi(i)) \in E_{\mu} \rightarrow i \in A_{\mu}
$$

suppose, contrary, that there is a $q \in N$ such that $f(\phi(i)) \in E_{q}$ but $i \notin A_{q}$.

Now, if $i \notin \mathrm{U}_{\mu=0}^{\infty} A_{\mu}, \Omega_{i}$ consists of empty sets only, and (2.8) gives $f(\phi(i)) \notin \bigcup_{\mu=0}^{\infty} E_{\mu}-\mathrm{a}$ contradiction. So, there is an $s \in N$ such that $i \in A_{s}$. By the first part of the proof we obtain $f(\phi(i)) \in E_{s}$. As $E_{s} \cap E_{q}$ $=\varnothing$ for $q \neq s$, it follows $s=q$.

So we have proved

$$
i \in A_{\mu} \leftrightarrow \psi(i) \in E_{\mu}
$$

i.e. that $A$ is r. reducible to $E$. 
TheOREM 3.2. If the creative sequence $A=A_{0}, A_{1}, \cdots$, is reducible to $B=B_{0}, B_{1}, \cdots$, then $B$ is a creative sequence.

Proof. Let $A$ be creative under $p$. Therefore, for every disjoint r.e. sequence $w_{h(\mu)}, \mu=0,1, \cdots$, satisfying $A_{\mu} \cap w_{h(\mu)}=\varnothing$ for all $\mu$, if $x \in I(h)$ then

$$
p(x) \notin \bigcup_{\mu=0}^{\infty}\left(w_{h(\mu)} \cup A_{\mu}\right) .
$$

If $f$ reduces $A$ to $B$ then

$$
A_{\mu}=f^{-1}\left(B_{\mu}\right), \quad \mu=0,1, \cdots .
$$

Denote by $\psi$ the $r$. function such that for all $x \in N$

$$
w_{\psi(x)}=f^{-1}\left(w_{x}\right) .
$$

There is a recursive function $\phi$ such that if $x \in I(F)$ then $\phi(x)$ $\in I(\psi(F)$ ) (the operation of composition being effective). We shall prove that $B$ is creative under $\chi=f(p(\phi))$.

Let $w_{k(0)}, w_{k(1)}, \cdots$, be any disjoint r.e. sequence of r.e. sets such that

$$
w_{k(\mu)} \cap B_{\mu}=\varnothing \quad \text { for all } \mu,
$$

and let $x$ be an index of the r. function $k$. We have to prove

$$
\chi(x) \notin \bigcup_{\mu=0}^{\infty}\left(w_{k(\mu)} \cup B_{\mu}\right) .
$$

By (3.9), using (3.7) and (3.8), we have

$$
A_{\mu} \cap w_{\psi(k(\mu))}=\varnothing, \quad \text { for all } \mu .
$$

As $A$ is creative and as $\phi(x) \in I(\psi(k))$, we get by (3.6)

$$
p(\phi(x)) \notin \bigcup_{\mu=0}^{\infty}\left(A_{\mu} \cup w_{\psi(k(\mu))}\right) .
$$

From (3.7), (3.8) and (3.12) follows now (3.10).

CoRollary 3.2.1. If a sequence $A$ is weakly creative it is creative.

Proof. Every creative sequence is reducible to $A$ by Theorem 3.1. By Theorem 3.2, $A$ is creative.

Theorem 3.3. If a weakly creative sequence $A=A_{0}, A_{1}, \cdots$, is 1-1 reducible to $B=B_{0}, B_{1}, \cdots$, then $B$ is a weakly creative sequence.

Proof. Let $A$ be weakly creative under $\phi$ and let the 1-1 r. function 
$f$ reduce $A$ to $B$. There is a recursive $\psi$ such that, for all $x \in N$,

$$
w_{\gamma(\mu, \psi(x))}=f^{-1}\left(w_{\gamma(\mu, x)}\right), \quad \mu=0,1, \cdots,
$$

(Take in (2.3) $Q(\mu, z, x, u) \leftrightarrow x \in f^{-1}\left(w_{\gamma(\mu, u)}\right) \wedge z=z$.)

Let $w_{\gamma(0, i)}, w_{\gamma(1, i)}, \cdots$, be a meager sequence. Then $w_{\gamma(0, \psi(i))}$, $w_{\gamma(1, \psi(i))}, \cdots$, is meager too.

Suppose first that $w_{\gamma\left(n_{0}, i\right)} \neq \varnothing$ and that $w_{\gamma\left(n_{0}, i\right)} \cap B_{n_{0}}=\varnothing$. Then $w_{\gamma\left(n_{0}, \psi(i)\right)} \cap A_{n_{0}}=\varnothing$ and, as $f$ is $1-1, w_{\gamma\left(n_{0}, \psi(i)\right)}$ is a singleton. Then $\phi(\psi(i)) \notin w_{\gamma\left(n_{0}, \psi(i)\right)}$ and, as

$$
y \in w_{\gamma\left(n_{0}, \psi(i)\right)} \leftrightarrow f(y) \in w_{\gamma\left(n_{0}, i\right)},
$$

we obtain $f(\phi(\psi(i))) \notin w_{\gamma\left(n_{0}, i\right)}$.

If all $w_{\gamma(\mu, i)}$ are empty, from $\phi(\psi(i)) \notin \bigcup_{\mu=0}^{\infty} A_{\mu}$ we obtain $f(\phi(\psi(i)))$ $\notin \bigcup_{\mu=0}^{\infty} B_{\mu}$.

This proves that $B$ is weakly creative under $f(\phi(\psi))$.

COROLlaRy 3.3.1. Every creative sequence is weakly creative.

Proof. By part (3) of Corollary 4 of Cleave's paper [1], every weakly creative sequence is $1-1 \mathrm{r}$. reducible to every creative sequence. By Theorem 3.3 follows the statement.

Corollaries 3.2.1 and 3.3.1 give

TheOREM 3.4. A sequence is weakly creative iff it is creative.

We point out that using the Definition 3.4 of the paper [2] of Lachlan one can give a definition of $M$-creativity (akin to Lachlan's definition of $M$-coproductivity) which is similar to our definition of weak creativity, but unnecessarily complicated. Namely, starting from the sequence $A=A_{0}, A_{1}, \cdots$, Lachlan constructs the sequence $A^{*}=A_{0}^{*}, A_{1}^{*}, \cdots$, where

$$
\begin{aligned}
A_{\mu}^{*} & =A_{\mu} & & \text { if } A_{\mu} \text { is a singleton, } \\
& =\varnothing & & \text { otherwise. }
\end{aligned}
$$

With this definition, $A$ will be called $M$-creative under $f$ iff $A$ is a r.e. sequence of r.e. sets and iff for all $i$

$$
\bigcup_{\mu=0}^{\infty}\left(W_{i, \mu} \cap A_{\mu}^{*}\right)=\varnothing \rightarrow\left\{f(i) \text { is defined and } W_{i, f(i)}=A_{i}=\varnothing\right\} .
$$

( $W_{i, j}$ is as in $\S 1$.) As $M$-creativity is equivalent with creativity it is equivalent with weak creativity.

On the ground of the Theorem 3.4 one can propose the following 
definition of creativity, which we shall call $S$-creativity:

A disjoint r.e. sequence $A=A_{0}, A_{1}, \cdots$, of r.e. sets is $S$-creative under a recursive $f$ iff for every disjoint sequence $w_{\gamma(0, i)}, w_{\gamma(1, i)}, \cdots$, for which $A_{\mu} \cap w_{\gamma(\mu, i)}=\varnothing$ for all $\mu$, we have

$$
f(i) \notin \bigcup_{\mu=0}^{\infty}\left(A_{\mu} \cup w_{\gamma(\mu, i)}\right) .
$$

It is not difficult to prove that a sequence is $S$-creative iff it is creative. The implication " $S$-creative $\rightarrow$ creative" is trivial. The converse implication is obtained through a theorem, similar to Theorem 3.3.

\section{REFERENCES}

1. J. P. Cleave, Creative functions, Z. Math. Logik Grundlagen Math. 7 (1961), 205-212.

2. A. H. Lachlan, Standard classes of recursively enumerable sets, Z. Math. Logik Grundlagen Math. 10 (1964), 23-42.

3. - Effective inseparability for sequences of sets, Proc. Amer. Math. Soc. 16 (1965), 647-653.

4. R. M. Smullyan, Theory of formal systems, Annals of Mathematics Studies No. 47, Princeton Univ. Press, Princeton, N. J., 1961.

University of Notre Dame 